ARTIGO

Recebido em: $17 / 05 / 2016$

Aceito em: 10/04/2017

\title{
Mapeamento tecnológico de patentes relacionadas a gamificação
}

\author{
Technological mapping of patents related to gamification
}

\author{
Claudia MENEZES (claudia.cardinale7@gmail.com)* \\ Robélius BORTOLI (robelius@yahoo.com.br)** \\ Claudio Pessôa de ALMEIDA (clapesalm@gmail.com)*** \\ * Doutoranda em Ciência da Propriedade Intelectual - UFS. \\ ** Professor do Departamento de Educação Física da UFS. \\ *** Mestrando em Ciência da Propriedade Intelectual - UFS.
}

\begin{abstract}
Resumo
Juntamente com a crescente popularidade dos jogos de vídeo, surgiu a gamificação para defender a extensão e aplicação de elementos normalmente presentes em jogos de vídeo para o mundo real e em áreas muito longe de apenas entretenimento. A gamificação é um fenômeno sócio tecnológico com potencial reivindicado para fornecer uma infinidade de benefícios relacionados a diversão, como também, benefícios sociais através de comunidades e interação social. Objetivo: Verificar os avanços e pesquisas tecnológicas que estão sendo realizadas sobre a gamificação por intermédio de análise de prospecção tecnológica. Uma vez que com o avanço acelerado do sistema de inovação, faz necessário a verificação do panorama mundial dos documentos de patentes relacionados a tecnologia da gamificação se atentando aos pedidos de depósitos em bancos de dados. Resultados: Constatou-se que as pesquisas relacionadas a gamificação são realizadas de forma recente e crescente. 0 dinamismo da tecnologia faz com que cada vez mais novos sistemas e métodos sejam desenvolvidas. Conclusão: Sendo assim o mapeamento tecnológico proporcionou verificar os avanços e pesquisas que estão sendo realizadas sobre a gamificação.
\end{abstract}

Palavras chave: Gamificação; Mapeamento Tecnológico; Patentes

\begin{abstract}
Along with the growing popularity of video games, gamification has emerged to advocate the extension and application of elements commonly found in video games to the real world and in areas far from just entertainment. Gambling is a socio-technological phenomenon with potential claimed to provide a plethora of fun-related benefits as well as social benefits through communities and social interaction. Objective: To verify the advances and technological researches that being carried out on the gamification by means of analysis of technological prospection. Since with the accelerated advancement of the innovation system, it is necessary to verify the world panorama of patent documents related to gamma technology by paying attention to requests for deposits in databases. Results: Found that the research related to recent form are held gamification and growing. The dynamism of technology causes more and more new systems and methods are developed. Conclusion: the technological mapping provided check advances and research that are performing about gamification.
\end{abstract}

Keywords: Gamification; Technological Mapping; Patents

v. 22, n. $49,2017$. p. $33-41$

ISSN 1518-2924

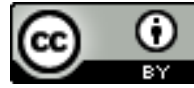

Esta obra está licenciada sob uma Licença Creative Commons 


\section{INTRODUCÃo}

Juntamente com a crescente popularidade dos jogos de vídeo, surgiu um movimento para defender a extensão e aplicação de elementos normalmente presentes em jogos de vídeo para o mundo real e em áreas muito longe de apenas entretenimento. A gamificação corresponde ao uso de mecanismos de jogos orientados para o objetivo de resolver problemas práticos ou para despertar engajamento entre um público específico. 0 desenvolvimento da gamificação, provém de uma constatação um tanto óbvia: seres humanos sentem-se fortemente atraídos por jogos (VIANNA et al., 2013). E quando empregado corretamente, a gamificação proporciona uma experiência inerentemente envolvente e, mais importante ainda, promove aprendizagem.

A definição de qualquer conceito é sempre uma tarefa difícil, a gamificação não é uma exceção, é definida por diferentes perspectivas, por vezes, em termos de comportamento, outras vezes em termos de predisposições, tendências, como processo de tratamento de informação, ou ainda, em termos de situação da aprendizagem. Assim, iniciaremos a definição da gamificação, considerando a expansão da indústria dos jogos que levou a dois desenvolvimentos paralelos: a Gamificação, indo além do que tradicionalmente são considerados como jogos e implementação de design do jogo; e a utilização do game design em diversas áreas (KOIVISTO e HAMARI, 2014).

No primeiro desenvolvimento define-se a partir dos autores considerados percussores, Prensky (2001) e Gee (2003). Gee descreve o impacto do jogo sobre o desenvolvimento cognitivo e identifica princípios de aprendizagem que poderiam ser encontrados em jogos de vídeo, ou seja, destacou o potencial de jogos de vídeo em processos de aprendizagem. Prensky (2001) também, defende o uso dos jogos eletrônicos no ensino, sugerindo que seu uso seria natural para as futuras gerações, criando a expressão de "nativos digitais" (SIMÕES; REDONDO; VILAS, 2013). No segundo desenvolvimento destaca-se a definição dos autores Liu, Alexandrova e Nakajima, (2011), onde potencial da utilização de jogos de vídeo ganhou a atenção de autores de diversas áreas e o objetivo máximo da gamificação é incentivar o usuário em situações não relacionados a jogos a ter o chamado comportamento de jogador: foco na tarefa em mãos, realizar várias tarefas ao mesmo tempo sob pressão, trabalhar a mais sem descontentamento, sempre tentar novamente quando falhar. Assim, entendemos que a gamificação não pode ser baseada apenas como um conjunto de métodos ou mecânica, mas além disso, deve ser entendida de forma mais abrangente, como um processo no qual o gamificar estar a tentar aumentar a probabilidade para as experiências gameful, imbuindo para o efeito o serviço com affordance (HUOTARI e HAMARI, 2012).

Atualmente, observa-se que a gamificação é mais frequentemente aplicada na área do marketing no nível dos sistemas de informação, assim a gamificação compreende o projeto e operação de pacotes de serviços gamified para a implementação de transformados e recém-criado processos de negócio (BLOHM e LEIMEISTER, 2013). Muitos autores relacionam o termo gamificação com outras abordagens comum aos videogames em aspectos não relacionados a jogos, é o caso dos serious games (jogos educativos). Porém, ressaltamos que os chamados serious games diferem da gamificação principalmente por serem jogos completos, utilizados para propósitos diferentes de apenas entretenimento. Enquanto as aplicações gamificadas não são jogos, apenas utilizam elementos de jogos para alcançar objetivos específicos (ALVES et al., 2012).

Outros autores relacionam a gamificação com os chamados jogos persuasivos. De acordo com Bergeron (2006), jogos são orientados para um público e uma área de influência específica, assim a premissa principal para a categorização deste gênero é a de que videogames são ao mesmo tempo mídias expressivas e persuasivas. No caso da gamificação pode ser considerada como uma tecnologia 
persuasiva, porém não se trata de jogos persuasivos completos e sim utilização de elementos de jogos, de acordo com a definição de Blohm e Leimeister, $(2013$, p. 2) “... uma tecnologia persuasiva que tenta influenciar o comportamento do usuário ativando motivos individuais via elementos game-design".

Assim, a gamificação passou a ser considerada como um fenômeno emergente, que deriva diretamente da popularização e popularidade dos games, e de suas capacidades intrínsecas de motivar a ação, resolver problemas e potencializar aprendizagens nas mais diversas áreas do conhecimento e da vida dos indivíduos. 0 objetivo desse estudo é verificar os avanços e pesquisas tecnológicas que estão sendo realizadas sobre a gamificação por intermédio de análise de prospecção tecnológica, considerando: a evolução anual dos depósitos de patentes, os países e escritórios dos depósitos dos documentos de patentes, os documentos de patentes por tipo de depositante, a frequência dos pedidos de patentes de acordo com a classificação internacional de patentes e por fim quantitativo de patentes de gamificação concedidas por áreas do conhecimento. Uma vez que, com o avanço acelerado do sistema de inovação, faz-se necessário a verificação do panorama mundial dos documentos de patentes relacionados a tecnologia da gamificação se atentando aos pedidos de depósitos em bancos de dados.

\section{METODOLOGIA}

O escopo metodológico desse artigo se resumiu ao mapeamento tecnológico de patentes relacionadas a gamificação. Em linhas gerais, uma patente é um direito exclusivo garantido por lei aos inventores ou titulares, por um período limitado, de monopólio das invenções. Em contrapartida, o detentor do invento concorda em revelar detalhes sobre a mesma, como por exemplo, informações prévias, natureza dos problemas técnicos solucionados pela invenção, uma descrição detalhada da invenção e do seu funcionamento e os desenhos quando necessários (THOMSON, 2016).

Segundo Teixeira e Souza (2013) documentos de patente podem ser considerados como fontes privilegiadas para o monitoramento do estado-da-arte, em termos de tecnologia, uma fonte direta de indicativos para processos de inteligência competitiva e monitoramento da concorrência. Nessa perspectiva, o levantamento das patentes foi realizado na base de dados Derwent Innovations IndexSM, uma base de dados de patentes da Thomson Reuters ${ }^{\circledR}$, integrada a plataforma Web of Knowledge® que cobre mais de 14,3 milhões de invenções, de 40 autoridades de emissões de patentes em todo o mundo, desde o ano de 1963.

0 mapeamento foi realizado seguindo os passos metodológicos fundamentais identificados por Rickinson e May (2009): escopo, busca, seleção, análise, síntese e elaboração de relatórios. 0 primeiro desses passos (escopo) culminou na definição sobre (a) o tema da pesquisa a ser adotada, ou seja, <gamif*> (gamificação, gamify, gamified, etc.); (b) a latitude da pesquisa refere-se ao (título de publicação), isto significava que os trabalhos selecionados para análise seriam aqueles que lidam com o tema gamificação diretamente em seus títulos como uma preocupação de investigação própria, ao contrário daqueles que simplesmente mencionam o tema por algum motivo; (c) o corte temporal dos trabalhos científicos publicados foi entre os anos de 1963 a 2015.

As informações não analisadas pela própria base de dados foram extraídas para o Microsoft Office Excel, e os dados foram classificados e selecionados de acordo anos de pedido das patentes, países de depósito, tipos de depositantes, depositantes de acordo a quantidade de patentes solicitadas, inventores de acordo com a quantidade de pedidos de proteção, quantidades de patentes de acordo com Classificação Internacional de Patentes (IPC, na sigla em inglês) e, por fim, número de patentes de acordo com as áreas de conhecimento. Para facilitar a análise e 
representação das informações foram geradas figuras gráficas, apresentadas e discutidas na seção seguinte.

\section{RESULTADOS E DISCUSSÃO}

A busca realizada na base de dados Derwent Innovations IndexSM resultou em 43 documentos de patentes referentes ao escopo metodológico, no entanto, é importante ressaltar que devido aos 18 meses de sigilo antes da publicação das solicitações de patentes, esse número poderá não corresponder ao número real de pedidos, principalmente para os anos de 2014 e 2015, uma vez que a pesquisa foi realizada em setembro de 2016. Assim, provavelmente novas tecnologias foram solicitadas proteção, porém, não foram computadas nesta pesquisa devido ao período de sigilo.

A figura 1 apresenta a evolução anual dos pedidos das patentes relacionados a gamificação, sendo constatado depósitos no período de 2013 a 2015. Comparando 2013 e 2014 houve um acréscimo de quase 50\% em patentes concedidas. Como a base Derwent não disponibiliza diretamente a informação sobre o ano de depósito da patente, utilizou-se a data de publicação da patente.

$\mathrm{O}$ ano de 2015 se destaca como o ano de maior quantidade de depósitos. 0 país depositante com maior frequência é os Estados Unidos com 33 depósitos. A primeira patente depositada diretamente nos Estados Unidos data de 28 de março de 2013, protocolada sob os números US2013079128-A1; US8821272-B2 e se refere a uma reivindicação independente que está incluído para um sistema de gamificação de eventos da vida real através da rede informatizada, intitulado "Método de gamificação de eventos da vida real através da rede informatizada, envolve a criação de instrução que diz respeito à utilização dos recursos da vida real pelo processador de módulo de tradução G-a-R, e é derivado de interação"; depositado pelos nomes e códigos dos depositantes da patente: THOMAS AJ (THOM-individual) DUDLEY J (DUDL-individual).

Atualmente foram detectados 43 depósitos de patentes relacionados a gamificação. Apesar de que o termo "gamificação" tenha sido empregado desde 2003 como um modo para influenciar o comportamento de usuários do mundo virtual e do mundo real, por meio de aplicações de software ou aplicativos móveis que incentivam as pessoas a realizarem uma variedade de atividades com recompensas para os usuários que exibem "certos" comportamentos (DALE, 2014). Surpreendentemente as primeiras patentes concedidas (figura 1), surgiram há apenas 3 anos, observa-se que trata de uma tecnologia recente e crescente.

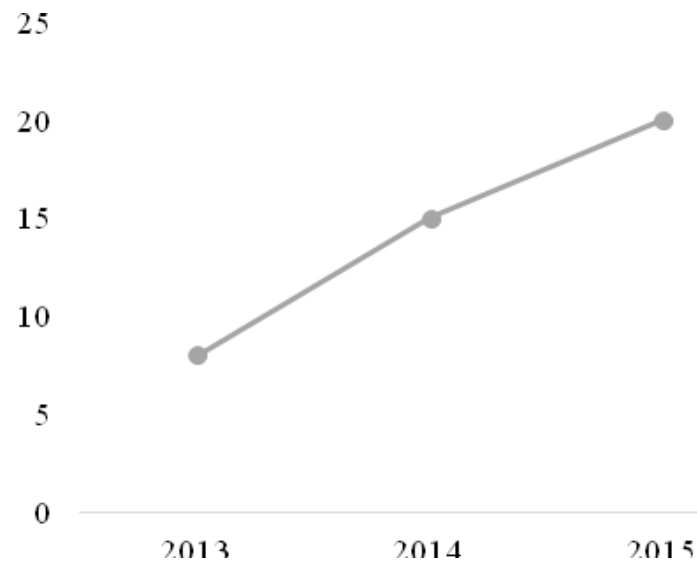

Figura 1: Evolução anual dos depósitos de patentes de gamificação Fonte: Derwent Innovations Index, 2016. Elaboração dos autores. 
A figura 2 apresenta os países depositantes de patentes envolvendo a gamificação, os dados foram extraídos e analisados a partir das bases Derwent Innovations Index. Ao analisar a origem dos pedidos de patentes, considerando a prioridade do documento, ou seja, o primeiro país de depósito do pedido de patente de invenção ou modelo de utilidade, se destacam, conforme figura 2, os escritórios dos Estados Unidos (77\%), Austrália (7\%) e m seguida os escritórios da República da Coréia e World Intellectual Property Organization (WIPO) (5\%), esta representa as patentes depositadas pelo Tratado de Cooperação de Patentes (PCT), ou seja, mediante a apresentação de um pedido de patente internacional sob o PCT, os candidatos podem procurar simultaneamente a proteção de uma invenção na maioria dos países do mundo (WIPO, 2017).

Isso indica que, embora a gamificação é compreensivelmente forte em sua "terra natal", os Estados Unidos da América, não deixa de ser uma área de interesse internacional para outros países. Nos EUA é característica do país, a ação de suporte indireto do Governo, principalmente as políticas de compra do Ministério da Defesa, que propiciaram, e propiciam, as condições para das Industria de Jogos Digitais. $\mathrm{Na}$ Coréia foram as políticas de estímulo ao desenvolvimento da Tecnologia da Informação e Comunicação (TIC) que estabeleceram as bases para o avanço da indústria de games (FLEURY, NAKANO e CORDEIRO, 2014).

Verifica-se, portanto a existência de uma concentração da Pesquisa e Desenvolvimento (P\&D) para tecnologias aplicadas a gamificação nos Estados Unidos. Vale ressaltar que no caso dos países que assinaram a Convenção da União de Paris (CUP), o artigo 52, parágrafo 2, exclui as patentes dos programas de computador. Assim, para os Estados-membros dessa legislação, os programas de computador "como softwares" não são definidos como sendo invenções e, consequentemente, não podem ser patenteados (REMICHE, 2005), justificando assim, a ausência destes países.

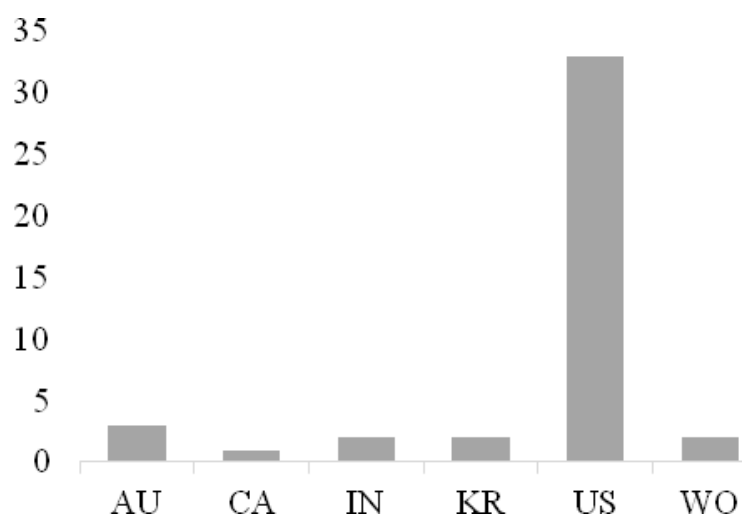

Figura 2: Países e escritórios dos depósitos dos documentos de patentes da gamificação Fonte: Derwent Innovations Index, 2016. Elaboração dos autores.

Legenda: $\mathrm{AU}=$ Australia, $\mathrm{CA}=$ Canada, $\mathrm{IN}=$ India, KR= Republic of Korea, US= United States of America, $\mathrm{WO}=$ World Intellectual Property Organization.

A figura 3 demostra a preeminência do setor empresarial no desenvolvimento de tecnologias para a gamificação, tendo requerido $55,81 \%$ das patentes, em seguida destacam-se os inventores independentes com 44,18\% pedidos de patentes. Algumas das empresas que depositaram são a Quest 2 Excel Inc, Salesforce.Com Inc, Sap Ag, Google Inc, Oracle Int Corp. 


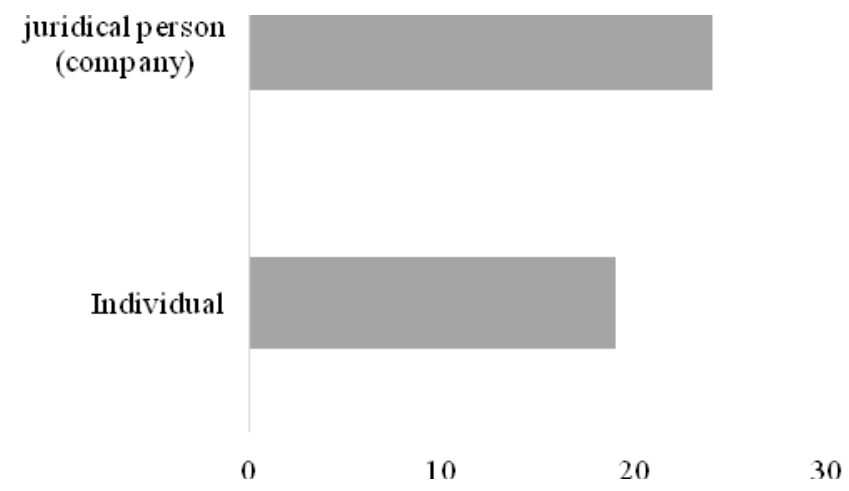

Figura 3: Documentos de patentes por tipo de depositante Fonte: Derwent Innovations Index, 2016. Elaboração dos autores.

Quanto às classificações de acordo com International Patent Classification (IPC) pode ser visto na figura 4, que a classe com maior ocorrência é a G06Q, que trata de sistemas ou métodos especialmente adaptados para propósitos administrativos, comerciais, financeiros, de gerenciamento, supervisão ou predição, seguidos por A63F que trata de jogos de cartas, mesa ou roleta, jogos em recintos fechados usando pequenas peças móveis para jogo e videogames e G06F que trata de processamento elétrico de dados digitais. Entre as com menos depósitos ficaram G02B que trata elementos, sistemas ou aparelhos ópticos, G06K que trata da identificação de dados; apresentação de dados; suporte de dados e manipulação de transportes de dados e G06N que trata de sistemas de computador baseado em modelos computacionais específicos.

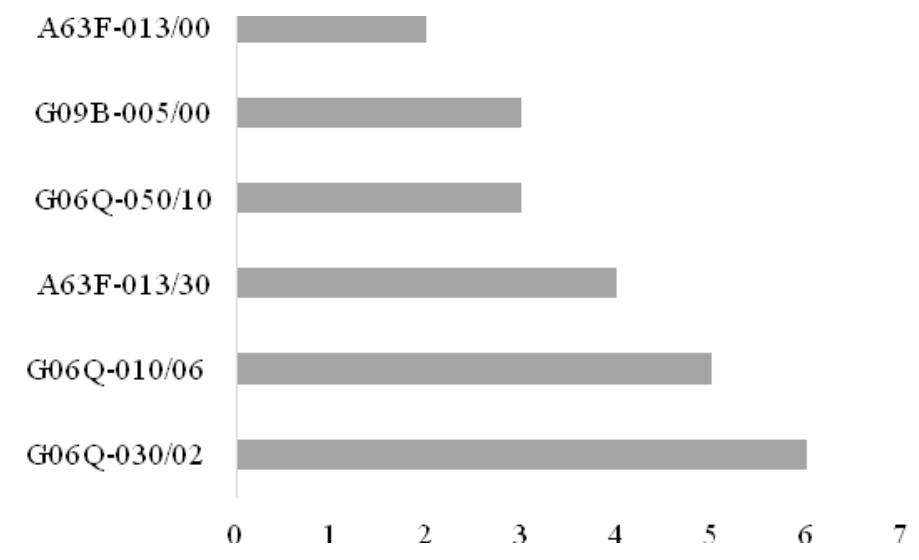

Figura 4: Frequência das classificações IPC nos Pedidos de patentes para Gamificação Fonte: Derwent Innovations Index, 2016. Elaboração dos autores.

Legenda:

A63F-013/00 Video games, i.e. games using an electronically generated display having two or more dimensions

G09B-005/00 Electrically-operated educational appliances

G06Q-050/10 Systems or methods specially adapted for a specific business sector, e.g. utilities or tourism/services

A63F-013/30 Interconnection arrangements between game servers and game devices; Interconnection arrangements between game devices; Interconnection arrangements between game servers 
G06Q-010/06 Resources, workflows, human or project management, e.g. organizing, planning, scheduling or allocating time, human or machine resources; Enterprise planning; Organizational models

G06Q-030/02 Marketing, e.g. market research and analysis, surveying, promotions, advertising, buyer profiling, customer management or rewards; Price estimation or determination

Esse destaque para a classe G06Q deve-se a tendência de onde a gamificação surgiu, dentro dos setores comerciais e de marketing (tais como cartões de pontos e recompensas associações, estruturas educativas, e produtividade no local de trabalho) e recentemente ganhou a atenção de acadêmicos, educadores e de profissionais das mais diversas áreas (SEABORN e FELS, 2015). Segundo Blohm e Leimeister (2013), o primeiro domínio da gamificação é na área do marketing a fim de melhorar a lealdade do cliente e imagem de marca. 0 apoio dos processos de aprendizagem é um segundo domínio do aplicativo principal de gamification.

Ao partir para a análise das áreas de conhecimento que mais utilizam depositaram patente de gamificação, à primeira vista, a amostra representada pela figura 5 abrange uma ampla gama de áreas, porém, a gamificação encontra na Ciência da Computação e na Engenharia áreas bastantes férteis para a sua aplicação. Nossa explicação especulativa para o destaque da Ciência da Computação, segue a afirmação de Caponetto, Earp e Ott (2014), que para a adoção da gamificação eficaz implica tanto determinada infraestrutura tecnológica, juntamente com um quadro de instrutores adequados. Assim é natural que a aplicação antecipada de gamificação destaque-se principalmente na área da Ciência da Computação, uma vez que possui a população de instrutores que tem as habilidades necessárias para a criação, adaptação e/ou a manutenção de infraestrutura tecnológica de suporte adequado para implementação de softwares gamificados.

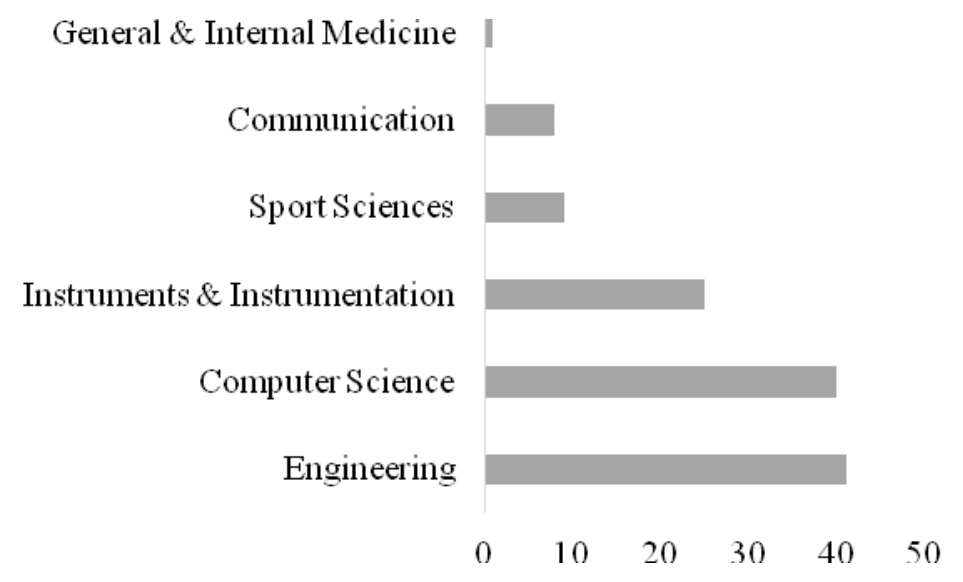

Figura 5 - Patentes de Gamificação por áreas do conhecimento

Fonte: Derwent Innovations Index, 2016. Elaboração dos autores.

\section{CONSIDERAÇÕES FINAIS}

Como é o caso de um conceito emergente, definir o termo "corretamente" é tanto desafiador e evasivo, porque depende de diferentes pontos de vista: fornecedores de plataforma, game designers, profissionais, observadores da indústria, etc. A manifestação do termo precede o conceito inicial da gamificação, embora a sua propagação só foi possível após o amadurecimento do seu conceito, com sua primeira patente dez anos após seu surgimento. Atualmente, a gamificação pode ser considerada uma nova tecnologia que incorpora elementos dos jogos em situações de não jogos, usada para envolver os clientes, estudantes e usuários na 
realização de tarefas cotidianas com recompensas e outros motivadores; definitivamente um efeito digno de exploração para todas as áreas.

Os resultados indicam que, embora a gamificação é compreensivelmente forte em seu "berço", os EUA, não deixa de ser uma área de interesse internacional na comunidade de pesquisa e tecnológica. No Brasil, vários anos mais tarde, somente agora as mídias sociais estão começando a aceitar a gamificação como um componente fundamental de aprendizagem organizacional e para o desenvolvimento de redes de conhecimento. O estudo indica ainda que a gamificação abrange uma ampla gama de áreas, porém, encontra na Ciência da Computação e na Engenharia áreas bastantes férteis para a sua aplicação.

Observou-se que empresas e pesquisadores individuais estão investindo na utilização da gamificação e isso raramente acontece, uma tendência com impacto em muitas áreas de negócio/sociedade. Assim, precisa-se compreender este mercado para ter uma apreciação mais profunda do potencial da gamificação e, concluindo, podemos prospectar que esta não é apenas uma moda passageira. A gamificação é uma nova tecnologia.

\section{REFERÊNCIAS}

BERGERON, Bryan. Developing serious games (game development series). 2006.

BLOHM, Ivo; LEIMEISTER, Jan Marco. Design of IT-based enhancing services for motivational support and behavioral change. Business \& Information Systems Engineering, p. 275-278, 2013.

CAPONETTO, Ilaria; EARP, Jeffrey; OTT, Michela. Gamificação and education: A literature review. In: ECGBL 2014: Eighth European Conference on Games Based Learning. p. 50-57. 2014.

DALE, Steve. Gamificação Making work fun, or making fun of work?.Business Information Review, v. 31, n. 2, p. 82-90, 2014.

FLEURY, Afonso; NAKANO, Davi; CORDEIRO, José Henrique Dell'Osso. Mapeamento da Indústria Brasileira e Global de Jogos Digitais. Santa Catarina: USC, 2014.

GEE, James Paul. What video games have to teach us about learning and literacy. Computers in Entertainment (CIE), v. 1, n. 1, p. 20-20, 2003.

HUOTARI, Kai; HAMARI, Juho. Defining gamificação: a service marketing perspective. In: Proceeding of the 16th International Academic MindTrek Conference. ACM. p. 17-22, 2012

KOIVISTO, Jonna; HAMARI, Juho. Demographic differences in perceived benefits from gamificação. Computers in Human Behavior, v. 35, p. 179-188, 2014.

LIU, Yefeng; ALEXANDROVA, Todorka; NAKAJIMA, Tatsuo. Gamifying intelligent environments. In: Proceedings of the 2011 international ACM workshop on Ubiquitous meta user interfaces. ACM, p. 7-12, 2011.

PRENSKY, Marc; PRENSKY, Mark. Digital game-based learning. St. Paul, MN: Paragon house, 2007.

REMICHE, Bernard. Revolução Tecnológica, Globalização e Direito de patentes. In Propriedade Intelectual e Desenvolvimento. Eds Varella, Marcelo Dias. Ann São Paulo: Lex Editora, p. 75-122, 2005.

RICKINSON, Mark; MAY, Helen. A comparative study of methodological approaches to reviewing literature. The Higher Education Academy, York, 2009. 
SEABORN, Katie; FELS, Deborah I. Gamificação in theory and action: A survey. International Journal of Human-Computer Studies, v. 74, p. 14-31, 2015.

TEIXEIRA, Renata Cristina; SOUZA, Renato Rocha. 0 uso das informações contidas em documentos de patentes nas práticas de Inteligência Competitiva:

apresentação de um estudo das patentes da UFMG. Perspectivas em Ciência da Informação, v. 18, n. 1, p. 106-125, 2013.

THOMSON CORPORATION. Derwent Innovations IndexSM 4.0 Seminar. Disponível em: <http://ip-

science.thomsonreuters.com/m/pt/dii4_sem_0104_po.pdf>. Acesso em: 29 jan.2016.

VIANNA, Ysmar et al. Gamificação Inc.: como reinventar empresas a partir de jogos. 2013.

WIPO. World Intellectual Property Organization. Disponível em http://www.wipo.int/pct/pt/. Acesso em: 10 jan. 2018. 